\title{
Linear versus Mel Frequency Cepstral Coefficients for Speaker Recognition
}

\author{
Xinhui Zhou $^{\# 1}$, Daniel Garcia-Romero ${ }^{\# 2}$, Ramani Duraiswami ${ }^{* 3}$, Carol Espy-Wilson ${ }^{\# 4}$, Shihab Shamma ${ }^{\# 5}$

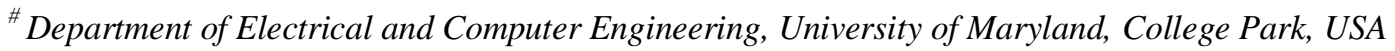 \\ ${ }^{1}$ zxinhui@umd.edu, ${ }^{2}$ dgromero@umd.edu, ${ }^{4}$ espy@umd.edu, ${ }^{5}$ sas@umd.edu \\ *Department of Computer Science, University of Maryland, College Park, USA \\ ${ }^{3}$ ramani@umiacs. umd. edu
}

\begin{abstract}
Mel-frequency cepstral coefficients (MFCC) have been dominantly used in speaker recognition as well as in speech recognition. However, based on theories in speech production, some speaker characteristics associated with the structure of the vocal tract, particularly the vocal tract length, are reflected more in the high frequency range of speech. This insight suggests that a linear scale in frequency may provide some advantages in speaker recognition over the mel scale. Based on two state-of-theart speaker recognition back-end systems (one Joint Factor Analysis system and one Probabilistic Linear Discriminant Analysis system), this study compares the performances between MFCC and LFCC (Linear frequency cepstral coefficients) in the NIST SRE (Speaker Recognition Evaluation) 2010 extended-core task. Our results in SRE10 show that, while they are complementary to each other, LFCC consistently outperforms MFCC, mainly due to its better performance in the female trials. This can be explained by the relatively shorter vocal tract in females and the resulting higher formant frequencies in speech. LFCC benefits more in female speech by better capturing the spectral characteristics in the high frequency region. In addition, our results show some advantage of LFCC over MFCC in reverberant speech. $\mathrm{LFCC}$ is as robust as MFCC in the babble noise, but not in the white noise. It is concluded that LFCC should be more widely used, at least for the female trials, by the mainstream of the speaker recognition community.
\end{abstract}

\section{INTRODUCTION}

\section{A. Motivation}

Mel-frequency cepstral coefficients (MFCC) [1] have been dominantly used in speaker recognition as well as in speech recognition. This is counterintuitive to many researchers since speech recognition and speaker recognition seek different types of information from speech, namely, phonetic information for speech recognition and speaker information for speaker recognition. MFCC was first proposed for speech recognition and its mel-warped frequency scale is to mimic how human ears process sound. Its spectral resolution becomes lower as the frequency increases. Therefore, the information in the higher frequency region is down-sampled by the mel scale. However, based on theory in speech production [2][3], speaker characteristics associated with the structure of the vocal tract, particularly the vocal tract length, are reflected more in the high frequency region of speech. This is illustrated in Fig. 1. Fig. 1a shows the schematic of a vocal tract. Fig. 1b shows a uniform tube as a vocal tract model
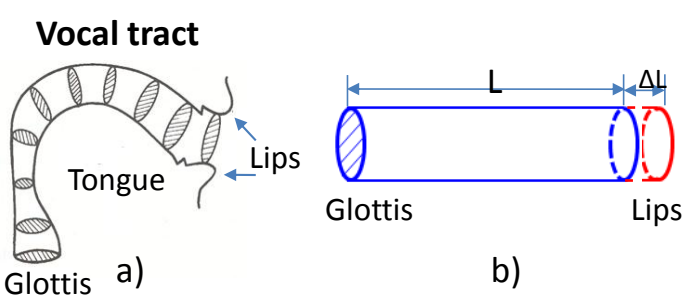

b)

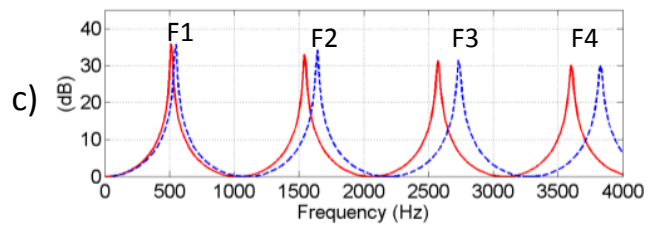

Fig. 1. A) Schematic of vocal tract. B) Simple tube model for vowel production (a schwa sound). C) Vocal tract acoustic responses for a length $\mathrm{L} 16 \mathrm{~cm}$ (solid) and length $17 \mathrm{~cm}$ $(\mathrm{L}+\Delta \mathrm{L})$.

for a schwa sound. Fig. 1c shows the acoustic responses of the tube at two different lengths. It can be seen that a $\Delta \mathrm{L}(1 \mathrm{~cm})$ change in the length leads to a much larger shift in F3 and F4 than in F1. The vocal tract length difference between male (average $17 \mathrm{~cm}$ ) and female (average $14 \mathrm{~cm}$ ) [2] makes the formant structures differ from each other significantly. This is the main reason why the trials between male and female in speaker recognition are less challenging and they are even excluded from the NIST SRE (Speaker Recognition Evaluation) [4].

Motivated by this insight from speech production, this study compares the performances between MFCC and linear frequency cepstral coefficients (LFCC) in speaker recognition. Fig. 2 shows an example of speech spectrum overlapped with both the mel filterbanks and the linear filerbanks. It can be seen that there are eleven linear filterbanks between F2 and F3, but only six mel filterbanks. We hope that, by capturing more spectral details in the high frequency region, the linear scale in frequency may provide some advantages in speaker recognition over the mel scale.

\section{B. Literature survey}

There are several studies in literature on comparing the speaker recognition performances of MFCC, LFCC, and other features and on finding an optimal frequency warping function. Based on the King speech database and GMM, [5] compared a 

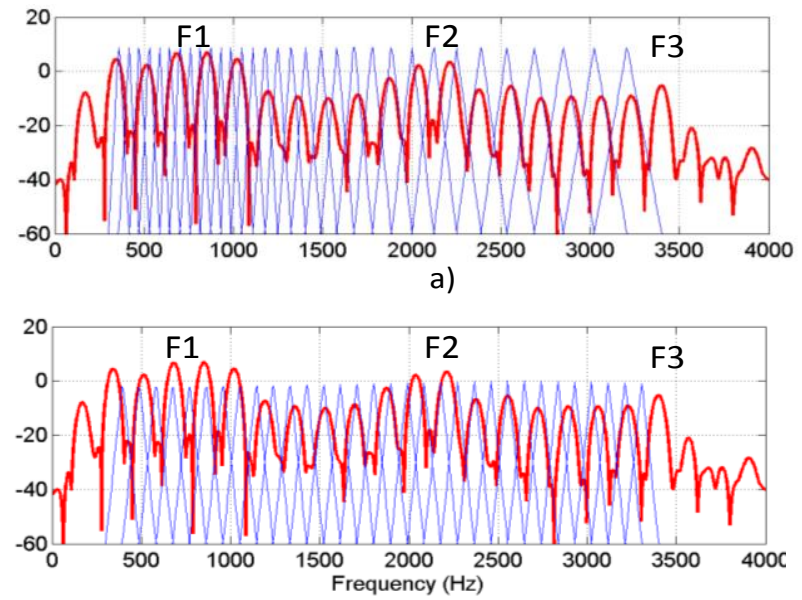

b)

Fig. 2. An example of speech spectrum overlapped with A) Mel filterbanks, and B) Liner filterbanks.

number of acoustic features in a speaker identification task, but no significant difference between MFCC and LFCC was found. Based on a small database with six males and five females and the dynamic-time-warping method, [6] showed some advantage of LFCC over MFCC. Based on two different criteria, [6] and [7] found that the optimal warping frequency for discriminating speaker lies between the mel scale and the linear scale. Both [6] and [7] reported a worse performance in LFCC than in MFCC even though the optimal functions obtained are closer to the linear scale. The speaker discriminative power in terms of F-ratio at different frequency bands $(0-8 \mathrm{kHz})$ was studied in [9]. It was on a small database including 23 males and 13 female speakers and a non-uniform warping function was obtained to outperform MFCC and LFCC in GMM for a speaker identification task. However LFCC has a worse performance than MFCC in four out of five conditions in [9]. MFCC, anti-MFCC and LFCC was tested in the NIST SRE06 in [10]. Based on a GMM-UBM system with factor analysis, [10] found that LFCC gave better performance only in nasal and nonnasal consonants, not in vowels. [11] used a modified set of LFCC for speaker identification in whisper speech and found LFCC is more robust to whisper speech. Very recently, [12] evaluated a number of acoustic features for speaker recognition using a classifier referred to as "GMM-Supervectors", and found that the LFCC improved accuracy over MFCC in five out of eight conditions on a private evaluation corpora sampled at $4 \mathrm{kHz}$. However, there is no further detailed analysis in [12] on how the accuracy was improved in those datasets by using LFCC.

Although there were efforts on comparing MFCC and LFCC, the results are inconsistent. This might be caused by different databases used, different classification methods, or even because of the different feature implementation, particularly when the source code is not accessible. However, to the best of our knowledge, no comparison study has been performed based on the current state-of-art speaker recognition systems. Furthermore, these studies only present the error rates, and no further analysis.

\section{Objectives}

The main objectives of this study are three-fold. First is to assess and compare the performances in speaker recognition between LFCC and MFCC on state-of-the-art back-end systems (the JFA system [13] and the PLDA system [14]). It is performed on the NIST SRE10 [4], the latest and presumably the most challenging NIST SRE task. Second is to further analyze the results and understand the underlying mechanism which accounts for our observations. Third is to evaluate the noise robustness of both features. Such a study will help us with our long-term goal, which is to find an optimal frequency-warping function for speaker recognition.

In the rest of this paper, we describe the experiment setups including the NIST SRE10 extended core task, the configurations for both MFCC and LFCC, and the two stateof-the-art back-end systems on which the SRE10 task is performed. Then we present and compare our results for MFCC and LFCC. In addition, their performances under noisy conditions are presented. Some explanations are provided for our observations. Finally, a summary along with our plans for future work are given.

\section{EXPERIMENT SETUP}

\section{A. NIST SRE10}

In the NIST SRE10 extended-core task, 21,595 English recordings from 236 female and 210 male speakers were collected from telephone conversations (Tel), telephone conversations recorded over a room microphone (Mic), and interview conversation recorded over a room microphone (Int). Some telephone conversations were in high- or low- vocal efforts. An average duration of 2.5 minutes of speech from the targeted speaker was present in the telephone conversations. The interview recordings were 3 to 15 minutes long. About 6.5 million trials were tested, each belonging to one of the following nine conditions: $\mathrm{C}$ : Int-Int same mic, C2: Int-Int different mic, C3: Int-Tel, C4: Int-Mic, C5: Tel-Tel, C6: TelTel-high vocal effort, C7: Mic-Mic-high vocal effort, C8: TelTel-low vocal effort, C9: Mic-Mic-low vocal effort. The trial number for each condition is presented in Table I, II, and III.

\section{B. Development data}

Our development data set comprised data from the NIST SRE 2004, 2005, 2006, and 2008 data sets, Switchboard-II, phases $2 \& 3$, Switchboard-Cellular part1\&2, and the Fisher database. A total of $23,904(31,655)$ telephone speech segments from 6,769 male (9,264 female) were obtained. For microphone recorded speech, 3,257 (4,183) segments from 203 male (257 female) speakers were obtained. For all the experiments, verification performance was reported in terms of equal error rate (EER) and/or the detection cost function (DCF) [4]. 

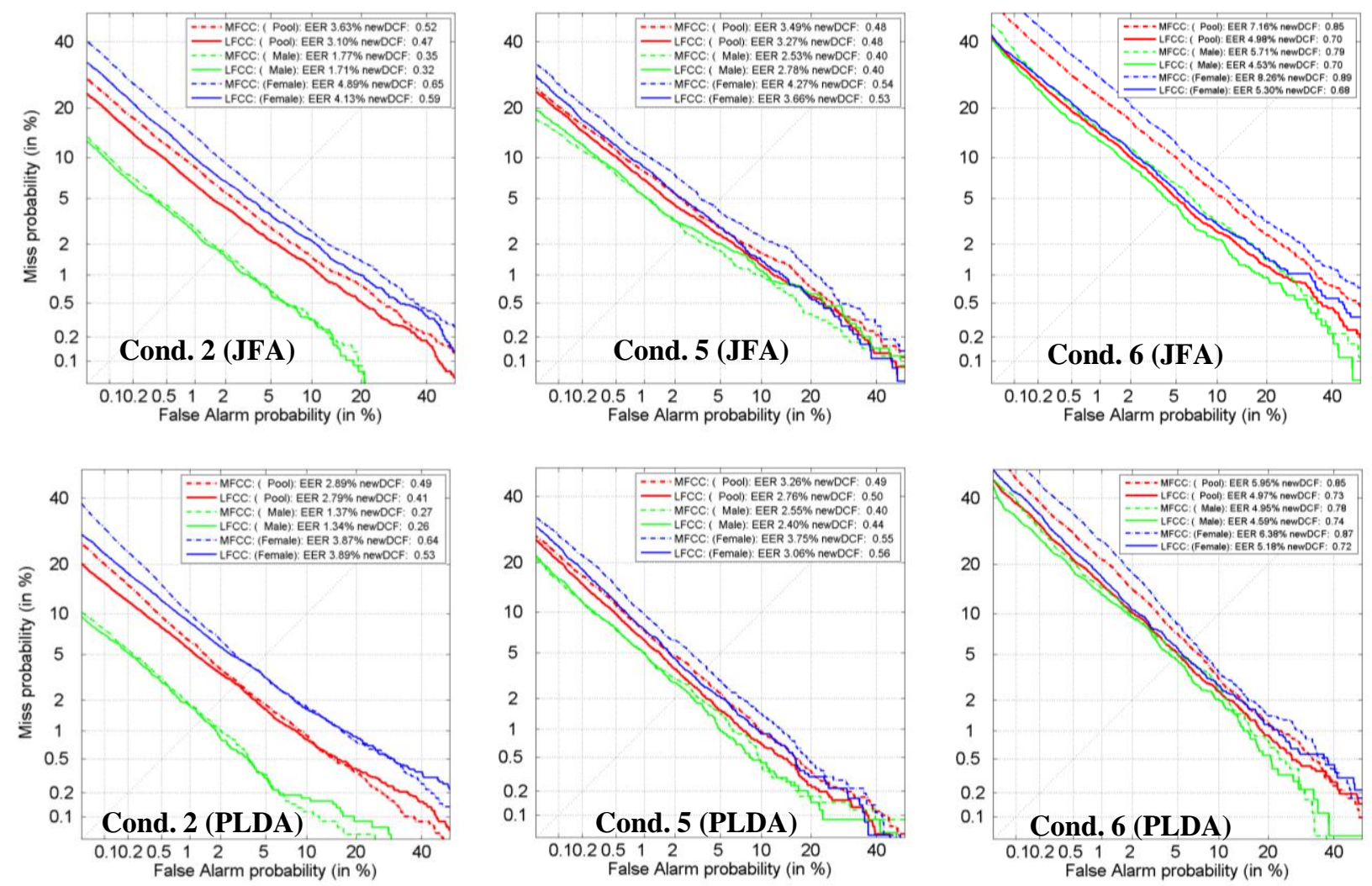

Fig. 3. DET (Detection Error Trade-off) curves for the conditions 2,5 and 6 in the NIST10 extended core task using MFCC and LFCC (Results for all trials (pooled), female and male trials shown in different colours, respectively, upper panel: JFA, lower panel: PLDA)

\section{Feature extraction}

A package named RASTAMAT [15] was adapted for extracting the MFCC and LFCC features ${ }^{1}$. The parameter configurations for both are the same except for the frequency warping scales. The speech signal is band-limited to 300-3400 Hz. 32 filter-banks were used, as shown in Fig. 2. The 19 cepstral coefficients plus its delta make a 38-dimensional feature vector. The analysis window is $20 \mathrm{~ms}$ with a shift of $10 \mathrm{~ms}$. The delta was performed over five frames.

The voice activity detection for segmenting speech from the silence region is based on the provided ASR transcript combined with the output of an energy-based VAD system. The cepstral mean subtraction and variance normalization was applied.

For the additive noise cases, only the region specified by VAD is counted for the signal-to-noise ratio. For reverberant speech, simulated room impulses at different reverberation times were used for creating reverberant speech.

\section{Two state-of-the-art back-end systems}

Below are the description of the two state-of-the-art backend systems we used in this study and their detailed descriptions are in [16][17].

\footnotetext{
${ }^{1}$ The MFCC/LFCC code is available online at http://www.glue.umd.edu/ zxinhui/LFCC_ASRU2011
}

The Joint Factor Analysis (JFA) system [16]: The JFA [13] paradigm provides an explicit mechanism to model the undesired variability in speech. Two separate genderdependent universal background models (UBM) with 2048 mixtures were trained using all of the development data. The JFA hyper-parameter sets were also gender-dependent. The eigenvoice and eigenchannel matrices $(\mathrm{V}$ and $\mathrm{U})$ were trained independently. First, a V matrix with 400 columns was trained by pooling together all the telephone and microphone recordings from the development set. Then, after projecting away the information in $\mathrm{V}$ from the supervectors, the residual term was used to train $\mathrm{U}=\left[\mathrm{U} \_\right.$tel U_mic ], with 100 columns from telephone data and 50 columns from microphone data. The residual matrix D was not trained and set to produce an equivalent relevance-MAP with a relevance factor of 16 [16].

The I-vectors and Probabilistic Linear Discriminant Analysis (PLDA) system [17]: Both the i-vector extractor as well as the PLDA systems were gender-dependent. BaumWelch sufficient statistics were collected using the same 2048 mixture UBMs as in the JFA system. The subspace matrix T with 400 columns was obtained by pooling together all the telephone and microphone recordings of the development set from the corresponding gender. For the PLDA model, the same data was used (excluding the Fisher database) to train the eigenvoice matrix $\Phi$ with 200 columns and the fullcovariance matrix $\Sigma \in \mathbb{R}^{400 \times 400}$. For all the experiments in additive noise and reverberation in this study, the PLDA scores were normalized using S-norm [17]. For the experiments with the original data, only the scores from trials 

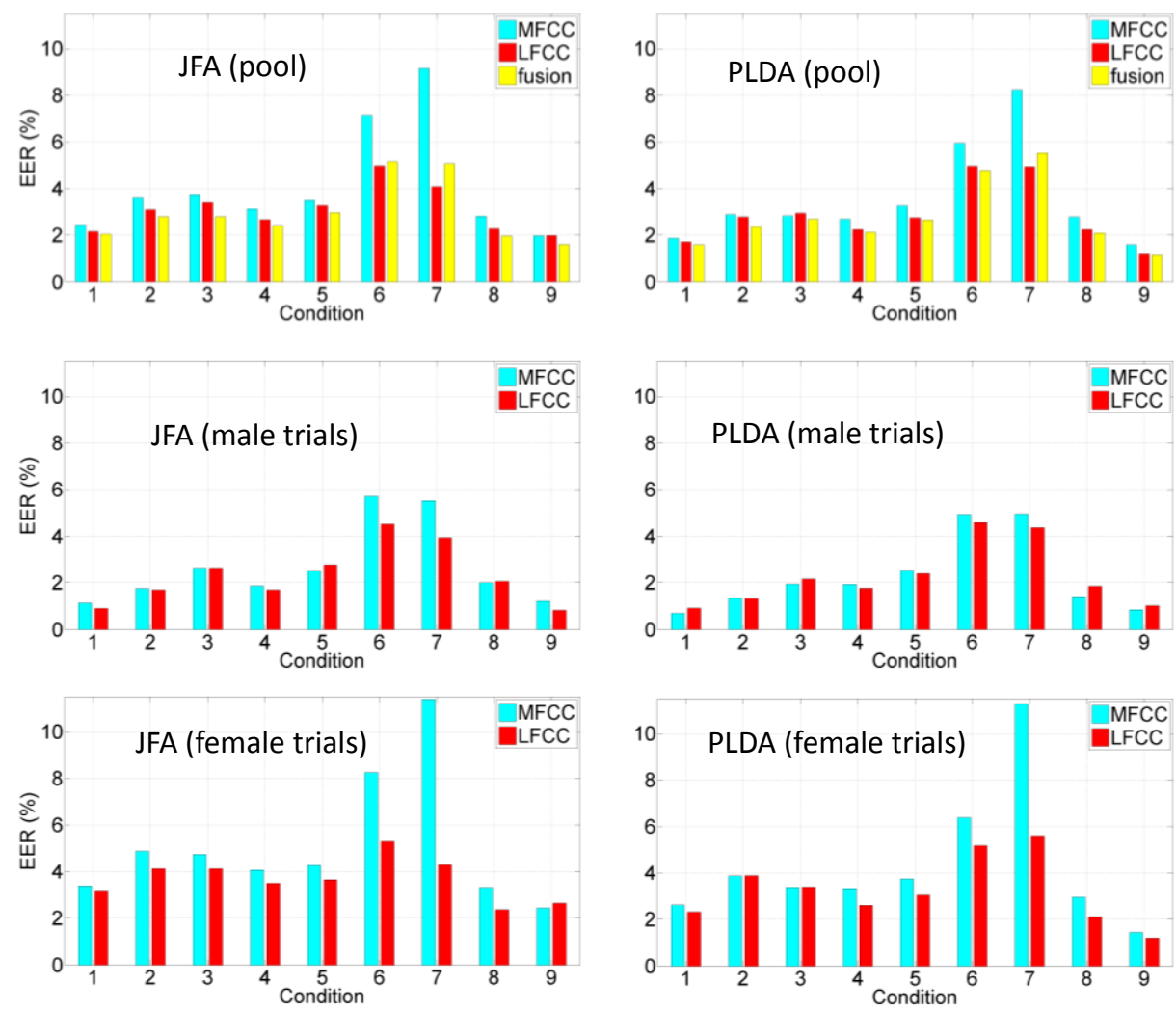

Fig. 4. EERs for the conditions 1-9 in the NIST SRE10 extended core task using MFCC and LFCC (Results for all the trials (pooled), female and male trials shown in different colours, respectively, fusion results are based on adding the scores of both MFCC and LFCC. Left panel: JFA, right panel: PLDA)

involving telephone speech were normalized since the performance was degraded by S-norm in conditions not involving telephone recordings.

\section{RESULTS}

\section{A. Performances of MFCC and LFCC in the SRE10 core- extended task}

To have a comprehensive performance comparison, the results of MFCC and LFCC on both JFA and PLDA are presented in Fig. 3, Fig. 4, and Tables I, II and III in different forms. Fig. 3 shows the DET curves. Fig. 4 shows the bar plots of EER, and Tables I-III show the EERs, the EER difference and the relative EER difference between MFCC and LFCC. In addition, they are presented for pooled, male, and female trials separately.

The DET curves for three conditions (2, 5 and 6 having the most number of target trials) are shown in Fig. 3. It can be seen that, in all the DET curves, the LFCC outperforms the MFCC for pooled trials (in red color). This is also demonstrated in terms of EER in the upper panel of Fig 4 and also Table I where LFCC outperforms MFCC in almost all the nine conditions for pooled trials (only two outliers with very small $\left.\Delta_{\mathrm{EER}}\right)$. It can be seen in the upper panel of Figure 4 and Table I that a simple additive score fusion can boost the performance in seven out of nine conditions. This indicates that, while LFCC outperforms MFCC in pooled trials, they are also complementary to each other.
It can be seen in Fig. 3 that the LFCC consistently outperforms MFCC in female trials, but the performances between them in male trials are much closer. This suggests that LFCC outperforms MFCC in pooled trials mainly due to its advantage in female trials. This advantage of LFCC in female trials can be seen in the lower panel of Fig. 4 and Table III in almost all the nine conditions on both back-end systems (outliers with very small $\Delta_{\mathrm{EER}}$ ). However, the advantage of LFCC over FMCC in male trials is not obvious. This advantage of LFCC in female trials can be explained by the relatively shorter vocal tract in female speakers and the resulting higher formant frequencies in their speech. LFCC benefits more in female speech by better capturing the spectral characteristics in the high formant frequency region.

Tables I, II, and III show all the EERs, the EER difference between MFCC and LFCC, and also the percentage relative to the EER of MFCC. It can be seen that the LFCC is a clear winner in pooled trials and in female trials.

\section{B. Performances of MFCC and LFCC in additive noise}

Fig. 5 shows the PLDA results of MFCC and LFCC in additive noise for all the trials in Condition 5, and the trends we observed are similar on both genders. Due to the space limitations, only the results for the Condition 5 are presented here. In the case of the white noise at OdB SNR, compared to the clean case, the EER is increased by $14.4 \%$ for MFCC, and by $16.6 \%$ for LFCC. In the case of babble noise at $0 \mathrm{~dB}$ SNR, 
Table I. The EERs ( \%) for all the trials (female and male trials are pooled) in conditions 1-9 in the NIST SRE10 extended core task using MFCC and LFCC. Results from both JFA and PLDA reported. Fusion results are based on adding the scores of both MFCC and LFCC, $\Delta_{\text {EER }}=E_{E E R} E_{L F C}-E_{\text {EFCC }}$ )

\begin{tabular}{|c|c|c|c|c|c|c|c|c|c|c|}
\hline \multicolumn{2}{|c|}{$\begin{array}{l}\text { Conditions } \\
\text { (TGT/NTGT trial number, } \\
\text { in thousands) } \\
\end{array}$} & $\begin{array}{c}\mathrm{C} 1 \\
(4.3 / 796.0)\end{array}$ & $\begin{array}{c}\mathrm{C} 2 \\
(15.1 / 2789.5)\end{array}$ & $\begin{array}{c}\text { C3 } \\
(4.0 / 637.9)\end{array}$ & $\begin{array}{c}\mathrm{C} 4 \\
(3.6 / 756.8)\end{array}$ & $\begin{array}{c}\text { C5 } \\
(7.2 / 409.0)\end{array}$ & $\begin{array}{c}\text { C6 } \\
(4.1 / 461.4)\end{array}$ & $\begin{array}{c}\mathrm{C} 7 \\
(0.4 / 82.6)\end{array}$ & $\begin{array}{c}\mathrm{C} 8 \\
(3.8 / 404.8)\end{array}$ & $\begin{array}{c}\text { C9 } \\
(0.3 / 70.5)\end{array}$ \\
\hline \multirow{4}{*}{ JFA } & MFCC(LFCC) & $2.46(2.17)$ & $3.63(3.10)$ & $3.77(3.41)$ & $3.14(2.68)$ & $3.49(3.27)$ & 7.16(4.98) & $9.16(4.09)$ & $2.82(2.28)$ & $1.98(2.00)$ \\
\hline & $\Delta_{\text {EER }}$ & -0.29 & -0.53 & -0.36 & -0.46 & -0.22 & -2.17 & -5.07 & -0.54 & 0.01 \\
\hline & $\Delta_{\mathrm{EER}} / \mathrm{EER}_{\mathrm{MFCC}}$ & $-11.8 \%$ & $-14.7 \%$ & $-9.5 \%$ & $-14.8 \%$ & $-6.2 \%$ & $-30.4 \%$ & $-55.4 \%$ & $-19.1 \%$ & $0.7 \%$ \\
\hline & Fusion & 2.05 & 2.80 & 2.81 & 2.43 & 2.98 & 5.17 & 5.08 & 1.96 & 1.61 \\
\hline \multirow{4}{*}{ PLDA } & MFCC(LFCC) & $1.88(1.73)$ & $2.89(2.79)$ & $2.84(2.96)$ & $2.70(2.25)$ & $3.26(2.76)$ & $5.95(4.97)$ & $8.25(4.95)$ & $2.79(2.25)$ & $1.60(1.20)$ \\
\hline & $\Delta_{\text {EER }}$ & -0.15 & -0.10 & 0.12 & -0.45 & -0.50 & -0.98 & -3.29 & -0.54 & -0.39 \\
\hline & $\Delta_{\mathrm{EER}} / \mathrm{EER}_{\mathrm{MFCC}}$ & $-8.1 \%$ & $-3.5 \%$ & $4.1 \%$ & $-16.6 \%$ & $-15.4 \%$ & $-16.5 \%$ & $-39.9 \%$ & $-19.4 \%$ & $-24.7 \%$ \\
\hline & Fusion & 1.61 & 2.36 & 2.69 & 2.13 & 2.66 & 4.80 & 5.53 & 2.10 & 1.15 \\
\hline
\end{tabular}

Table II. The EERs (\%) for the male trials

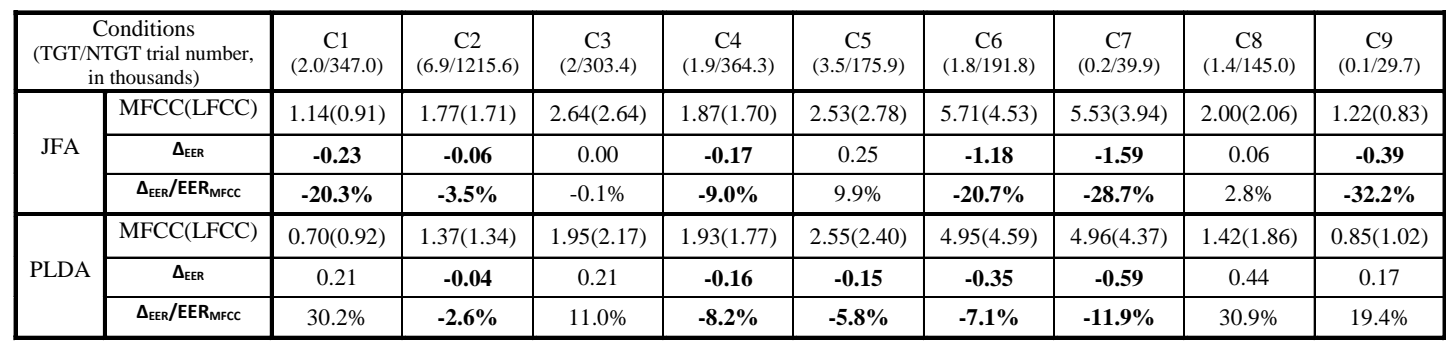

Table III. The EERs (\%) for the female trials

\begin{tabular}{|c|c|c|c|c|c|c|c|c|c|c|}
\hline \multicolumn{2}{|c|}{$\begin{array}{c}\text { Conditions } \\
\text { (TGT/NTGT trial number, } \\
\text { in thousands) }\end{array}$} & $\begin{array}{c}\mathrm{C} 1 \\
(2.3 / 449.1)\end{array}$ & $\begin{array}{c}\mathrm{C} 2 \\
(8.2 / 1573.9)\end{array}$ & $\begin{array}{c}\text { C3 } \\
(2.0 / 334.4)\end{array}$ & $\begin{array}{c}\mathrm{C} 4 \\
(1.8 / 392.5)\end{array}$ & $\begin{array}{c}\text { C5 } \\
(3.7 / 233.1)\end{array}$ & $\begin{array}{c}\text { C6 } \\
(2.3 / 269.7)\end{array}$ & $\begin{array}{c}\text { C7 } \\
(0.2 / 42.7)\end{array}$ & $\begin{array}{c}\text { C8 } \\
(2.4 / 260.0)\end{array}$ & $\begin{array}{c}\text { C9 } \\
(0.2 / 40.8)\end{array}$ \\
\hline \multirow{3}{*}{ JFA } & MFCC(LFCC) & $3.40(3.16)$ & $4.89(4.13)$ & $4.74(4.13)$ & $4.07(3.51)$ & $4.27(3.66)$ & $8.26(5.30)$ & $11.40(4.31)$ & $3.32(2.38)$ & $2.44(2.66)$ \\
\hline & $\Delta_{\mathrm{EER}}$ & -0.24 & -0.76 & -0.61 & -0.57 & -0.61 & -2.96 & -7.09 & -0.94 & 0.21 \\
\hline & $\Delta_{\text {EER }} /$ EER $_{\text {MFCC }}$ & $-7.1 \%$ & $-15.5 \%$ & $-12.9 \%$ & $-13.9 \%$ & $-14.3 \%$ & $-35.8 \%$ & $-62.2 \%$ & $-28.3 \%$ & $8.8 \%$ \\
\hline \multirow{3}{*}{ PLDA } & MFCC(LFCC) & $2.63(2.33)$ & $3.87(3.89)$ & $3.39(3.40)$ & $3.35(2.62)$ & $3.75(3.06)$ & $6.38(5.18)$ & $11.28(5.61)$ & $2.96(2.10)$ & $1.45(1.21)$ \\
\hline & $\Delta_{\text {EER }}$ & -0.30 & 0.02 & 0.01 & -0.72 & -0.70 & -1.20 & -5.67 & -0.86 & -0.24 \\
\hline & $\Delta_{\mathrm{EER}} / \mathrm{EER}_{\mathrm{MFCC}}$ & $-11.5 \%$ & $0.5 \%$ & $0.2 \%$ & $-21.7 \%$ & $-18.6 \%$ & $-18.8 \%$ & $-50.3 \%$ & $-29.0 \%$ & $-16.5 \%$ \\
\hline
\end{tabular}

compared to the clean case, the EER is increased by $10.6 \%$ for both MFCC and LFCC.

The results show that LFCC is as robust as MFCC in babble noise, but not in the white noise. The energy in the high frequency region of speech is usually weak and it is more susceptible to noise corruption. LFCC has more filterbanks in the high frequency region and this is why it is less robust in the white noise than MFCC.

\section{Performances of MFCC and $L F C C$ in reverberation}

Fig. 6 shows the PLDA results of MFCC and LFCC in the reverberant speech in condition 5 for all the trials, only male trials and only female trials, respectively. In the case of RT30 $500 \mathrm{~ms}$, compared to the clean case, the EER for female trials is increased by $12.1 \%$ for MFCC and by $9.8 \%$ for LFCC. In the case of RT30 $500 \mathrm{~ms}$, compared to the clean case, the EER for male trials is increased by $8.2 \%$ for MFCC and by $8.0 \%$ for LFCC. The results show some advantage of LFCC over MFCC in reverberant speech, but mainly for female trials.

One possible explanation for this advantage is the narrowbanded linear filter-bank [18]. The early reflection in a room impulse response is usually less than $25 \mathrm{~ms}$ and it can be captured by the narrow-banded linear filter-bank in the high frequency region and removed through the cepstral mean subtraction, whereas the mel filterbank in the high frequency region is broad-banded and does not have this property.

\section{DISCUSSIONS}

Our results in SRE 2010 Conditions 6 and 7 suggest that LFCC may have some advantages in vocal effort and is also consistent to the results in [11]. The intuition is that vocal efforts are related to the voice excitation and reflected in the low frequency region of speech. MFCC emphasizes this region, whereas LFCC does not. However, our observations in Conditions 6 and 7 were not repeated for male trials in the SRI-FRTIV corpus [19]. Only slight improvement in the male trials was obtained, so it does not confirm that LFCC helps in vocal effort, although the improvement of LFCC over MFCC was still observed in the female trials. Further analysis is needed to understand the potential advantage of LFCC on vocal effort.

In addition to the mel filterbank and the linear filterbank, the cochlear or auditory filterbank was also studied for speaker recognition by some researchers [12]. However, its performance is not as good as MFCC and LFCC [12]. One explanation is that the auditory filterbank center frequencies are in the tonotopic order and have even lower resolution in 

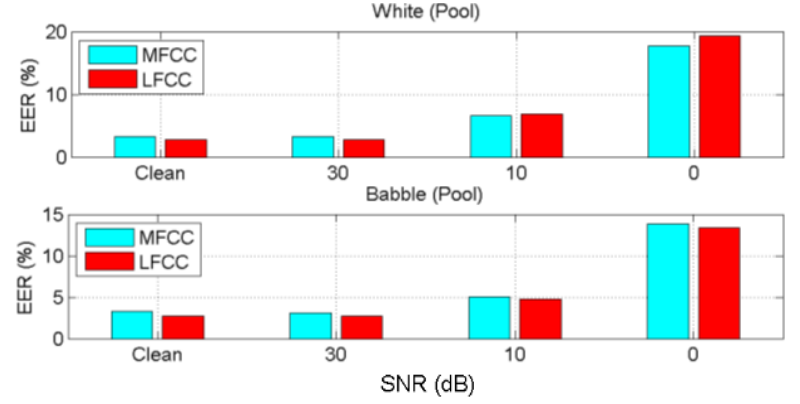

Fig. 5. EERs for condition 5 with artificial additive noise in the test data. (Upper panel: white noise, and Lower panel: babble, the results are for all the trials and are based on the PLDA)
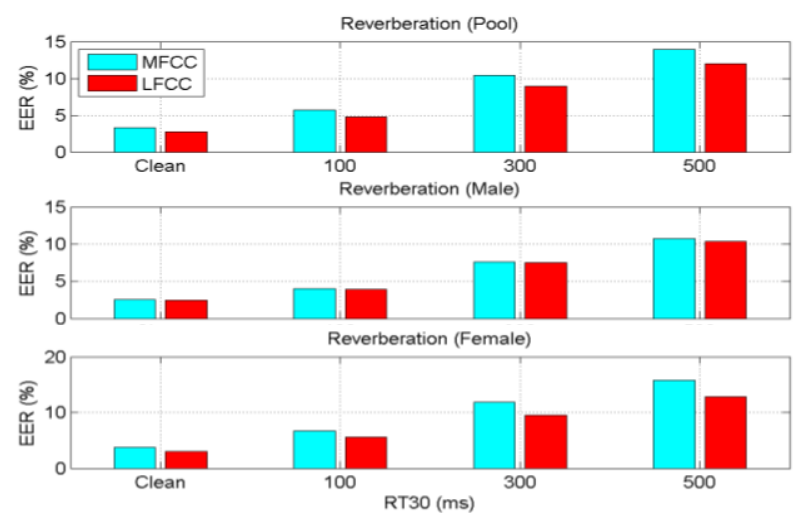

Fig. 6. EERs for condition 5 with artificial reverberation in the test data on the PLDA system. (Upper panel: all the trials,

Middle panel: male trials, and Lower panel: female trials)

the high frequency region than the mel filterbank. One possible strategy to enhance the performance of an auditory feature is to use a linear scale but integrate other processing procedures for additional benefits such as noise robustness.

\section{CONCLUSION}

This study was motivated by insight from speech production that some speaker characteristics associated with the structure of the vocal tract, particularly the vocal tract length, are reflected more in the high frequency region of speech. Based on two state-of-art speaker recognition backend systems, this study compares the performances between MFCC and LFCC in the NIST SRE 2010 extended-core task. Our results in SRE10 show that, while they are complementary to each other, LFCC consistently outperforms MFCC mainly due to its better performance in the female trials. This can be explained by the relatively shorter vocal tract in females and the resulting higher formant frequencies in speech. LFCC benefits more in female speech by better capturing the spectral characteristics in the high frequency region. In addition, our results show some advantage of LFCC over MFCC in reverberant speech. LFCC is as robust as MFCC in the babble noise, but not in the white noise. In conclusion, our results suggest that LFCC should be more widely used, at least for the female trials, by the mainstream of the speaker-recognition community.

Our current and future work aims to find an optimal frequency warping method (for male and female trials, respectively) for the speaker recognition task, and also to extend and test the linear frequency range to broadband instead of only in the telephone frequency band.

\section{ACKNOWLEDGMENT}

This research was partially funded by the Office of the Director of National Intelligence (ODNI) and Intelligence Advanced Research Projects Activity (IARPA) through the Army Research Laboratory (ARL), and by NSF award \#0917104. All statements of fact, opinion, or conclusions contained herein are those of the authors and should not be construed as representing the official views or policies of NSF, ODNI, the IARPA, or the U. S. Government. In addition, we would like to thank Dr. Dmitry Zotkin for help with some of the preliminary experiments for this work.

\section{REFERENCES}

[1] S. B. Davis and P. Mermelstein, "Comparison of parametric representations for monosyllabic word recognition in continuously spoken sentences, " IEEE Trans. ASSP, vol. 28, no. 4, 357-366, 1980

[2] K. N. Stevens, Acoustic phonetics. Cambridge, Mass.: MIT Press, 1998.

[3] Story, B.H., (2003). Using imaging and modeling techniques to understand the relation between vocal tract shape and acoustic characteristics, Proceedings of the Stockholm Music Acoustics Conference, 6-9 August.

[4] 2010 NIST Speaker Recognition Evaluation, http://www.itl.nist.gov/ iad/mig/tests/sre/2010/NIST_SRE10_evalplan.r6.pdf

[5] D. A. Reynolds, Experimental Evaluation of Features for Robust Speaker Identification, IEEE Transactions on Speech and Audio Processing, Vol. 2, No. 3, pp. 639-643, October 1994.

[6] M. Homayounpour and G. Chollet. A comparison of some relevant parametric representations for speaker verification. In Proc. ESCA Workshop on Automatic Speaker Recognition, Identification and Verification, 1994. 1-9.

[7] C. Miyajima, H. Watanabe, K. Tokuda, T. Kitamura, S. Katagiri, A new approach to designing a feature extractor in speaker identification based on discriminative feature extraction, Speech Communication, Volume 35, Issues 3-4, October 2001, Pages 203-218.

[8] R. Auckenthaler and J. Mason (1997), "Warping Function For Sub-Band Error Equalisation In Speaker Recognition", Workshop on Speaker Recognition and its Commercial and Forensic Applications ( RLA2C), page 194-197.

[9] X. Lu, J. Dang, "Physiological feature extraction for textindependent speaker identification using non-uniform subband processing", Proc. of ICASSP, 2007.

[10] H. Lei \& E. Lopez "Mel, Linear, and Antimel Frequency Cepstral Coefficients in Broad Phonetic Regions for Telephone Speaker Recognition", Interspeech 2009, Brighton, UK, 2009

[11] Xing Fan and John H.L. Hansen, "Speaker Identification with Whispered Speech based on modified LFCC Parameters and Feature Mapping", in ICASSP 2009, Taipei, Taiwan.

[12] A. Lawson, P. Vabishchevich, M. Huggins, P., Ardis, B. Battles, and A. Stauffer, "Survey and evaluation of acoustic features for speaker recognition", ICASSP 2011, pp.5444-5447.

[13] P. Kenny, N. Ouellet, N. Dehak, V. Gupta, and P. Dumouchel, "A study of interspeaker variability in speaker verification," IEEE Transactions on Audio, Speech, and Language Processing, 16:980-988, 2008.

[14] S. J. D. Prince and J. H. Elder, "Probabilistic linear discriminant analysis for inferences about identity," in Proc. ICCV'07, Rio de Janeiro, Brazil, Oct 2007, pp. 1-8.

[15] D. Ellis (2005), PLP and RASTA (and MFCC, and inversion) in Matlab, available online: http://www.ee.columbia.edu/ dpwe/resources /matlab/rastamat/.

[16] D. Garcia-Romero and C. Espy-Wilson, "Joint factor analysis for speaker recognition reinterpreted as signal coding using overcomplete dictionaries," Proc. Odyssey Speaker and Language Recognition Workshop, June 2010.

[17] D. Garcia-Romero and C. Espy-Wilson, "Analysis of I-vector Length Normalization in Speaker Recognition Systems", INTERSPEECH 2011 , Florence, Italy, pp.249-252.

[18] S. Thomas, S. Ganapathy, and H. Hermansky, "Recognition of Reverberant Speech Using Frequency Domain Linear Prediction," IEEE Signal Processing Letters, vol. 15, pp. 681-684, 2008.

[19] E. Shriberg, M. Graciarena, H. Bratt, A. Kathol, S. Kajarekar, H. Jameel, C. Richey, and F. Goodman,"Effects of vocal effort and speaking style on textindependent speaker verification," Proc. of Interspeech, Brisbane, Australia, 2008 